\title{
Construction duration estimation for advanced nuclear power plants
}

W. Robb Stewart, Jeremy Gregory, Koroush Shirvan

\section{Abstract}

Recent nuclear construction project schedules in the US and Europe were $2.5 x$ their original estimates due to productivity misses, supply chain challenges, hiring constraints, and other issues. Small modular reactors are proposed as a remedy. Smaller reactors will require one fourth to one third the personhours of labor and leverage more common supply chains, putting less strain on each step of the construction process. This study presents a methodology for estimating the construction duration of nuclear projects, so the sensitivity of proposed concepts to staffing and labor constraints can be analyzed. The methodology builds on a previous overnight capital cost estimation tool and builds a Gantt chart out of 226 tasks. The genetic algorithm assigns resources to each task to minimize construction time subject to peak staffing and hiring rate constraints. The results were consistently within $15 \%$ of seven benchmark durations. Under tighter labor constraints, the construction duration of large reactors increased $42 \%$ on average and only $3 \%$ for small reactors. Modularization of systems and structures reduced construction durations $60 \%$, but it increased the sensitivity to labor constraints. Small reactors were more sensitive to the modularization assumptions because of the compact structures and layout, and the power specific cost sensitivity was higher, but the total financial impact was smaller because the total costs were lower.

II.
\begin{tabular}{|l|l|}
\hline BWR & Acronyms \\
\hline DOE & Deparing water reactor \\
\hline EEDB & Economic of Energy \\
\hline GA & Genetic algorithm data base \\
\hline IDC & Interest during construction \\
\hline LWR & Light water reactor \\
\hline PWR & Pressurized water reactor \\
\hline SMR & Small modular reactor \\
\hline
\end{tabular}

\section{Introduction}

Capital construction costs for nuclear plants are $50-75 \%$ of lifetime costs [1], and interest during construction (IDC) can account for up to one third of total capital costs [2]. Recent nuclear projects in the US and Europe experienced significant construction delays and cost overruns increasing the importance of these financing costs [3]. A new wave of nuclear plant concepts is emerging [4], and many of these aim to mitigate the cost and construction challenges facing the industry, especially small modular reactors (SMRs). Stewart and Shirvan built an overnight capital cost estimation tool for nuclear power plants [5], and this work expands that tool to include estimates of the construction duration specific to the reactor architecture. In the same vein as the cost estimating tool, we aim to fill the gap observed by Mignacca et al., that "there is a lack of standardized approach in the evaluation and economic performance of SMRs" [6]. 
Several studies reviewed the historical relationship between construction duration and attributes of a nuclear plant. Berthélemy and Escobar Rangel found key factors correlating to lead times were future electricity demand, nuclear experience of the country and vendor, generating capacity of the plant, and whether the construction started before or after the accidents at Three Mile Island and Chernobyl (Berthélemy and Escobar Rangel, 2015). Similarly, Lovering et al. noted the importance of a stable regulatory environment to construction cost and duration [8]. In addition, reports from nongovernmental organizations highlight the importance of effective project management, supply chain maturity, labor productivity, and design maturity [9] and [3].

For new nuclear plant concepts, there are some public construction duration estimates. Vendors of Gen III+ light water reactors (LWRs), provided estimates to a US Department of Energy (DOE) funded report on large, advanced reactor designs [10]. These durations ranged from 54-61 months for site preparation to fuel loading. The study concluded that while aggressive, these durations were achievable given historical rates of concrete, steel, and piping installation. In a study of modularization, Maronati et al. estimated seven years for a stick-built Westinghouse SMR and four years for a modularized version [11]. Maronati et al. also estimated four years for the Integral Inherently Safe Light Water Reactor [12]. Neither of these estimates was associated with a detailed justification for the estimate. The Energy Impact Center, who is using an open-source design methodology, published a 29-month construction duration for their 100 MWe LWR SMR [13]. Their duration estimate comes from a Gantt chart with more than 100 activities making it one of the most detailed published durations. However, the labor constraint assumptions behind these estimates were not published, so they cannot be replicated or extrapolated to new architectures.

Independent estimates of construction durations for advanced nuclear are scarce. To estimate the construction time for SMRs, Abdulla et al. surveyed sixteen experts [14]. On average, these experts estimated that SMRs would take three years to build as opposed to five years for large reactors. To study the impact of modularization, Lloyd and Roulstone created a methodology to scale 120 construction activity durations based on data from the Sizewell B nuclear construction project. The method scaled activity durations and wait times between activities using power capacity [15]. Their results indicated that with full modularization, SMRs could be constructed in 3.5 years and large reactors in 5 years, in contrast to 5.1 and 6.4 years respectively without modularization. Their study was for a generic SMR and did not consider the intricacies of any specific reactor design.

The ABWR experience from GE-Hitachi provided interesting motivation for this study. Despite having the largest concrete, steel, and building volumes among Gen III and Gen III+ reactors [16], TEPCO constructed the first ABWR unit at the Kashiwazaki-Kariwa nuclear power plant in a record 40 months from site preparation to fuel loading [17]. This, in addition to the data from Lovering and Berthélemy and Escobar Rangel, break the notion that larger plants necessarily have longer construction durations. Modularization, open-top construction, and other advanced construction techniques played in a significant role in the ABWR experience [18].

This paper presents a methodology to estimate design-specific construction durations for advanced nuclear power plants including SMRs. The methodology then estimated the construction duration for several reactor architectures listed in Table 1. These architectures were based on commercial reactor plant designs but were not identical to them. We evaluated the impact of system modularization (including factor labor productivity an associated costs), structural modularization, and advanced 
construction techniques such as steel plate composites and very heavy lift cranes on total construction duration.

Table 1. Reactor architectures considered in this study

\begin{tabular}{|l|l|l|l|l|l|l|}
\hline Name: & $\begin{array}{l}\text { PWR12 } \\
\text { [Better Experience } \\
\text { (BE) and Median } \\
\text { Experience (MW) }\end{array}$ & $\begin{array}{l}\text { Large passive } \\
\text { safety PWR } \\
\text { (LPSR) }\end{array}$ & $\begin{array}{l}\text { Multi-module } \\
\text { natural } \\
\text { circulation } \\
\text { (MMNC) }\end{array}$ & $\begin{array}{l}\text { Natural } \\
\text { circulation } \\
\text { SMR } \\
\text { (NC-SMR) }\end{array}$ & $\begin{array}{l}\text { Large modular } \\
\text { BWR } \\
\text { (LM-BWR) }\end{array}$ & $\begin{array}{l}\text { Small } \\
\text { modular BWR } \\
\text { (SM-BWR) }\end{array}$ \\
\hline Similar to: & $\begin{array}{l}\text { Westinghouse 4- } \\
\text { Loop PWR }\end{array}$ & $\begin{array}{l}\text { Westinghouse } \\
\text { AP1000 }\end{array}$ & NuScale & $\begin{array}{l}\text { Holtec } \\
\text { SMR-160 }\end{array}$ & $\begin{array}{l}\text { GE-Hitachi } \\
\text { ABWR }\end{array}$ & $\begin{array}{l}\text { GE-Hitachi } \\
\text { BWRX-300 }\end{array}$ \\
\hline $\begin{array}{l}\text { Net power } \\
\text { (MWe): }\end{array}$ & 1144 & 1117 & $\begin{array}{l}12 \times 74 \\
(888 \text { total) }\end{array}$ & 160 & 1350 & 290 \\
\hline $\begin{array}{l}\text { Source } \\
\text { material }\end{array}$ & {$[19]$} & {$[20,21]$} & {$[22,23]$} & $124,25]$ & {$[26,27]$} & {$[28,29]$} \\
\hline $\begin{array}{l}\text { Total } \\
\text { concrete/ } \\
\text { MWe }\end{array}$ & 122 & 71 & 189 & 184 & 202 & 196 \\
\hline
\end{tabular}

\section{Methods}

To estimate the construction duration, we started from a list of 226 tasks and associated person-hours of labor to complete these tasks. The person-hours for each task were outputs of a nuclear capital cost estimation tool from Stewart and Shirvan that scaled reference costs from EEDB based on attributes of the reactor architecture [5]. A genetic algorithm (GA) searched for the duration and wait time for each task to minimize the total duration subject to constraints on resources, worker space, and task interdependency.

\section{A. Labor hours per task}

The Nuclear Cost Estimation Tool (NCET) of Stewart and Shirvan estimates the costs of over 200 structures, systems, and components (SSCs) by scaling a set of reference costs using estimates of concrete volume, pressure vessel mass, steam generator heat transfer area and other inputs [5]. The reference database for costs includes labor hours associated for the construction or installation of each SSC [19], and labor hours scale similarly to costs [15], so we applied the cost scaling factors from NCET to the reference labor hours.

We collected data on the SSC input parameters for each of the reactor architectures in Table 1 from publications and licensing documents on the similar commercial design. The supplementary information contains a complete set of the inputs to these scaling relationships, the parameters of the governing equations, and the resulting estimates of labor hours per task.

NCET makes several cost adjustments for modularization, learning-by-doing, advanced manufacturing, design simplicity, and passive safety where relevant for specific reactor architectures. The most relevant of these for this study were the assumptions on modularization. In being consistent with the onsite labor reduction estimates for modularization from McKinsey, modular systems moved half of the reference onsite labor to a factory thus removing it from the labor hours considered in estimating the construction duration [30]. The model assumed factory labor was twice as efficient as site labor, so only one half of the labor moved offsite was added to the factory cost. Moving the costs from the site labor category to the factory category increased the impact of the learning rate, lowered the impact on indirect costs, and as shown here, reduced the total construction duration. 


\section{B. Task dependencies}

In construction projects, most tasks cannot be started until a previous task has been completed, and these dependencies constrain the critical path. We used publicly available Gantt charts for nuclear projects from EEDB, the Energy Impact Center, and the Japan Atomic Energy Agency to assign each task a predecessor task $[13,19,31]$. In some cases, the predecessor tasks were required to be $100 \%$ complete before the new task could be started, but in other cases the predecessor task only needed to be 50,70 , or $90 \%$ complete. Further, each task was labeled as requiring a civil, mechanical, or electrical resource or some fractional combination. We assessed the validity of these dependency assumptions by comparing the staffing levels of civil, mechanical, and electrical staffing over time to published staffing levels from the International Atomic Energy Agency in the results section [32]. Table 2 has a sample of the task descriptions, the predecessor task, the required completion of the predecessor task, and the resource labeling, and the full set of 226 tasks is included in the supplemental information.

Table 2. Sample subset of the tasks. predecessor tasks, and resource labeling

\begin{tabular}{|l|l|l|r|r|r|r|}
\hline Account & Description & $\begin{array}{l}\text { Predecessor } \\
\text { task }\end{array}$ & $\begin{array}{l}\text { Predecessor task } \\
\text { required completion }\end{array}$ & Civil & Mechanical & Electrical \\
\hline A.211. & Site preparation & None & 0 & 1 & 0 & 0 \\
\hline A.212.13 & Reactor building substructure & A.211. & 0.5 & 1 & 0 & 0 \\
\hline A.212.141 & Reactor building superstructure & A.212.13 & 0.9 & 1 & 0 & 0 \\
\hline A.212.140 & Reactor building interior concrete & A.212.141 & 0.5 & 1 & 0 & 0 \\
\hline A.212.21 & Plumbing \& Drains & A.212.141 & 1.0 & 0 & 1 & 0 \\
\hline
\end{tabular}

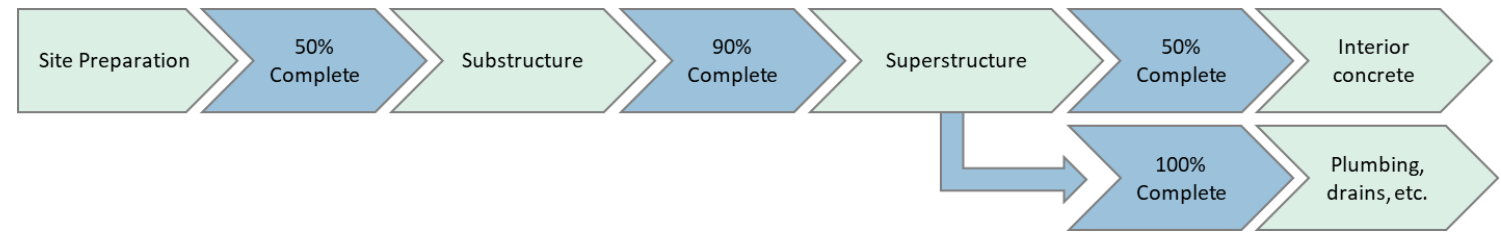

Figure 1. Graphical representation of the task - predecessor task dynamics from Table 2

To build the schedule, each task had a delay and task length parameter, both in months. To evaluate the schedule, the method steps through month long timesteps. At each timestep, all tasks whose predecessor tasks were completed to the required completion fraction were launched. Then, if applicable, the delay parameter could prevent immediate progress on the task for a number of months. The delay parameter allows for resource smoothing. After the launch and optional delay, a task was considered active. Active tasks were progressively completed in the following timesteps according to their durations. Figure 2 graphically portrays the schedule evaluation process for a single set of task, predecessor task, and delay. This process continued until all tasks were completed. The objective of the optimization problem was to find a delay and task length for each task that minimized the total construction duration subject to the constraints. This delay (or wait time) and active task work split was similar to an approach from Lloyd and Roulstone [15]. 


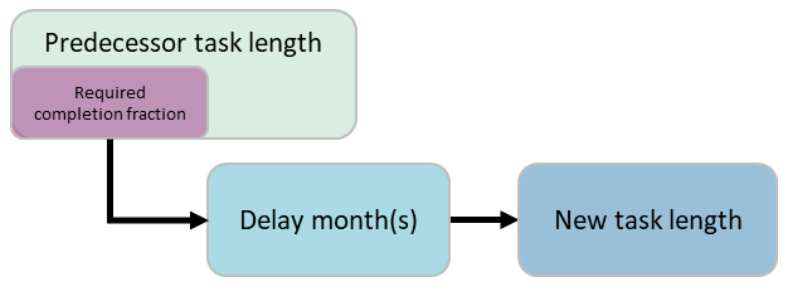

Figure 2. Schedule evaluation process for a single task and predecessor task, including the delay period

\section{Constraints}

One of the dominant constraints in the scheduling problem are resource constraints [33]. In the case of the nuclear construction process, these constraints were simplified to: maximum site staffing of 4500 , maximum monthly deployable labor rate of 800 , and a building staffing limit. Table 3 summarizes these constraints.

In practice, the maximum site staffing depends on the country and resource availability where the plant is to be constructed. For example, the Barakah nuclear plant had four reactors under construction with a total net capacity of $5.6 \mathrm{GWe}$, and the maximum onsite staffing was 18,000 workers or 3.2 workers/MWe [34]. In contrast, for the Hinkley Point C proposal, EDF forecasted peak staffing of 5,500 for two reactors with a net capacity of $3.3 \mathrm{GWe}$, or 1.7 workers/MWe [35]. The nature of the labor market and reactor architecture in Barakah allowed for a $90 \%$ higher per-MWe staffing level. In this study, all reactor architectures were resource constrained to a maximum site staffing upper bound of 4,500 direct-workers, i.e. not including management and supervision. We explored the sensitivity to this constraint in the results.

The lower bound constraint of 2,500 came from a normalized staffing profile from IAEA [32] that we scaled so that the integrated total labor hours matched the PWR12-BE in EEDB[19]. Both datasets had construction durations of 72 months and were 4-loop PWRs. Multi-unit sites are more likely to be governed by the upper bound constraint, but in this study, we only considered single-unit sites except in the case of the MMNC architecture which is inherently multi-unit.

The second constraint was the maximum monthly staffing change or the allowable increase or decrease in site staffing across a given month. This constraint also varied greatly across different sites. Multi-unit sites who could easily transfer resources from one project to the next were less constrained by the rate of recruiting and hiring new staff. The most recent, detailed publication of staffing ramp rates was the Application Summary Document for Hinkley Point C from EDF, and there the staffing profile showed a maximum monthly deployment of 800 workers [35]. In contrast, the IAEA staffing profile had no monthly changes greater than 160 workers. Therefore, we assumed an 800 upper bound and a 160 lower bound maximum monthly labor deployment to evaluate the sensitivity to this constraint.

The model calculated the staffing level for each task based on the required labor hours, the task length, and a 160 hour work-month. In the process of doing the analysis, some cases had difficulty converging with the 160-worker constraint due to the total person-hours sizes of the tasks and the uniform staffing assumption. To remedy this, the monthly staffing change constraint applied to a moving average with a three-month window for the 160 -worker constraint. The results from the 800 -worker constraint were insensitive to whether the moving average was applied or not. 
The final constraint limited the number of workers within a given building space. A key objective of this work was that the construction duration estimate would be sensitive to and reflective of the unique attributes of a reactor architecture. One aspect of accomplishing this goal was the design specific labor hour estimates coming from the NCET, and the second aspect was to capture the staffing limitations of each design. However, a second objective of the methodology was to remain generalizable and simple enough to apply to new architectures where detailed design information may be scarce. To strike this balance, we assigned every task to a building and applied a basic formula to constrain the number of workers inside at each timestep. The building assignments for each task are in the supplementary information, and the assignments varied across the reactor architectures to best describe each plant.

The formula for constraining the maximum workers for a building was a power law relationship:

$$
\text { Max workers }=A *(\text { building floor area })^{n}
$$

In most cases the building floor area came from our review of the buildings in licensing documents and other publications. This information is included in the supplementary information. To estimate the values of $A$ and $n$, we iteratively solved the optimization problem for the PWR12-ME and -BE reactors using different values of $A$ and $n$. The resulting approximation was $A=2.1$ and $n=0.7$. The validity of these approximations is demonstrated in the benchmarks in the results section.

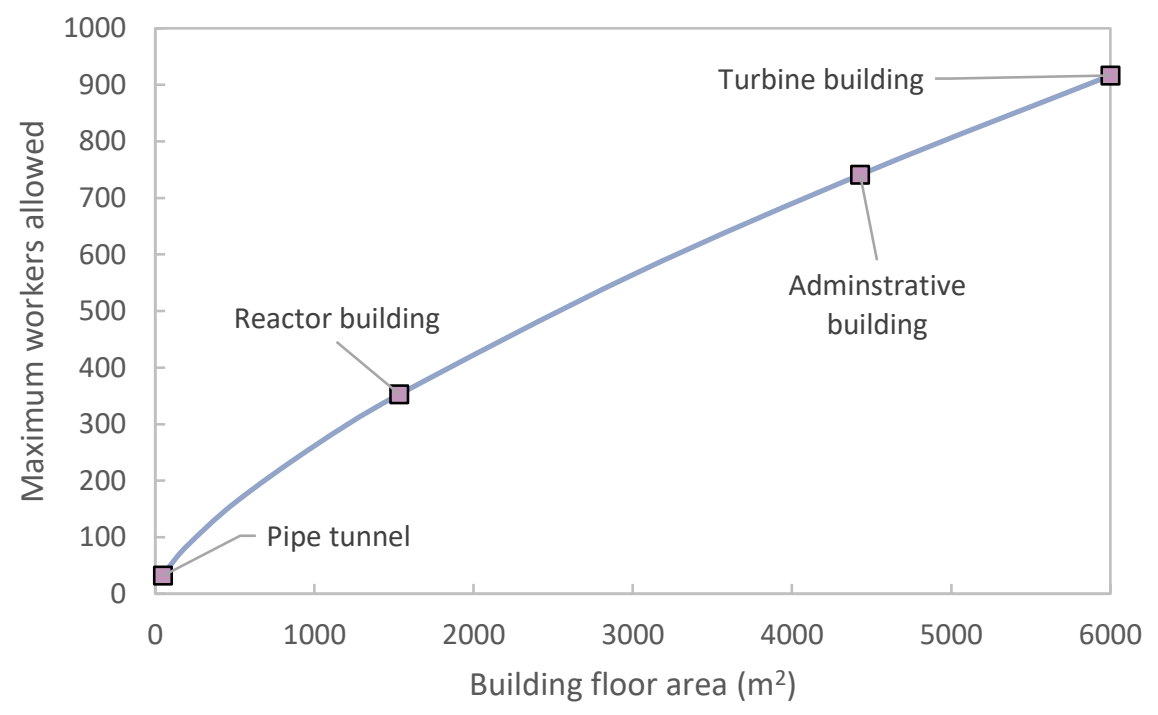

Figure 3. How the building maximum worker constraint scaled with building floor area. The data labels indicate the values for the PWR12 reference plant.

We applied each constraint using an exterior penalty function summing the magnitude of the violation of the constraint at each timestep and added to the objective function. In this way, the optimization algorithm was allowed to include infeasible solutions early in the evolution, but eventually all cases found a feasible solution. 
Table 3. Constraints as applied using exterior penalty functions.

\begin{tabular}{|l|c|c|}
\hline Constraint & Implementation & \\
\hline $\begin{array}{l}\text { Maximum site } \\
\text { staffing penalty }\end{array}$ & $\sum_{t=1}^{t=\text { end of last task }} \max \left(\right.$ staffing $\left._{\text {total }}-X, 0\right)$ & $X=\{2500,4500\}$ \\
\hline $\begin{array}{l}\text { Maximum } \\
\text { labor change } \\
\text { penalty }\end{array}$ & $\sum_{t=1}^{\text {t=end of last task }} \max \left(\left|\frac{d\left(\text { staffing }_{\text {total }}\right)}{d t}\right|-Y, 0\right)$ & $Y=\{160,800\}$ \\
\hline $\begin{array}{l}\text { Building } \\
\text { staffing limit } \\
\text { penalty }\end{array}$ & $\sum_{t=1}^{t=\text { end of last task }}\left[\sum_{n=1}^{n=\text { last building }} \max \left(\right.\right.$ staffing $_{n}-$ limit $\left.\left._{n}, 0\right)\right]$ & \\
\hline
\end{tabular}

\section{Advanced construction techniques}

In addition to modularization of SSCs moving onsite labor offsite, the other advanced construction technique considered in this study was very heavy lift cranes (VHLC) coupled with open-top construction. These two techniques were discussed in depth by the IAEA, and Figure 4 shows how the VHLC functions in practice [36]. VHLC and open-top construction allow for the parallelization of labor across the site - separate elements of a building can be fabricated in separate work locations and then lifted into place. Effectively, this relaxes the building staffing constraint by increasing the building work area [37]. To apply this construction technique, we increased the building area for the tasks associated with VHLC capable SSCs by a factor of three to represent two additional worksites adjacent to the primary structure.

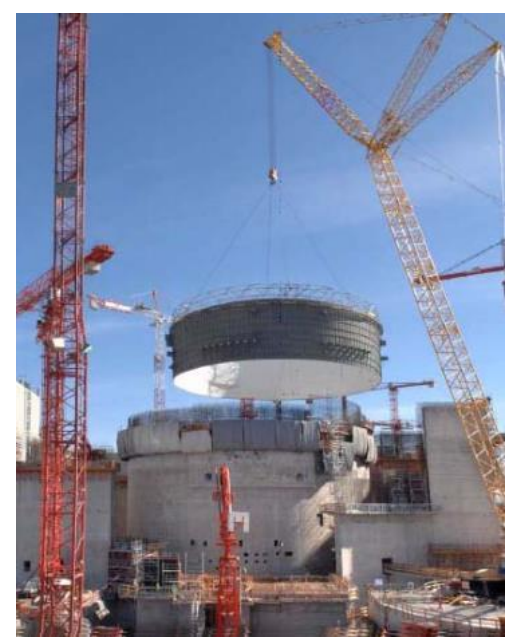

Figure 4. VHLC installing the containment liner for Olkiluoto 3 in Finland [32].

\section{E. Genetic algorithm: problem formulation}

\section{a) Primary formulation: full scale problem}

Heuristics, linear programming, and evolutionary methods have all been used to solve scheduling problems. Linear programming can find the exact optimal solution to unconstrained, simple scheduling problems, but realistic scheduling problems face constraints and a large number of decision variables which at best increases the computation time and at worst makes finding a feasible solution impossible. Defining the set of rules to govern the heuristic is complex and often not generalizable which was a key objective for this study. Therefore, many have turned to GAs to quickly solve large, constrained scheduling problems $[33,38]$. GAs stochastically evolve a population of solutions over many generations. In a given generation, the best individuals in the population, according to the objective function, are 
selected; then they are recombined with one other of the best solutions; finally, they are mutated, resulting in a new population of candidate solutions. This process is repeated until a maximum number of generations is reached or a set of convergence criteria is met.

The problem at hand had 452 decision variables, two per task: the delay and the task length. The large number of decision variables required a large population. We used the GA Python module, Pymoo, a multi-objective optimization framework, because it allows for vectorized objective functions [39]. Vectorizing the objective function resulted in dramatically shorter evaluation periods even with large populations. Our formulation adopted the default settings on crossover, mutation, and convergence from Pymoo. After Pymoo found an optimal solution for the PWR12-ME, future initial populations were pre-populated with this best solution to accelerate the convergence time.

After this full-scale GA converged, we passed the resulting optimal solution through a second optimization routine, called the subproblem GA. The full-scale GA found a near-optimal solution within about 300 generations, but the last generations progressed very slowly. When evaluating the entire schedule at once, changes to most decision variables would not yield any change to the objective function, and this slowed the evolution process because not all the schedule dynamics were exposed to the optimizer. To accelerate this process, we created a time-step based subproblem formulation. We reformulated the optimization problem to only change the parameters of the tasks which were launched but not active at each timestep starting from the first timestep and moving to the next timestep after converging, as shown in Figure 5. The subproblem tasks could either have just had their predecessor task reach the required completion fraction or they could be in their delay period. This reduced the number of decision variables from 452 to between 2 and 50, and the smaller set of decision variables greatly allowed the GA to find a more optimal solution more quickly. The process required looping through all timesteps 3-5 times before converging.

The primary limitation of the subproblem formulation was that it required a good "first guess" solution that was already feasible. Iterating on the parameters for a subset of the tasks when non-considered tasks were causing infeasibility made it difficult for this method to converge in some cases. However, when starting from a good initial guess, this formulation converged in 2-3 minutes. The process for the results in this study, unless otherwise noted, was to run the full-scale GA until convergence, and then run the subproblem GA starting from the full-scale GA.

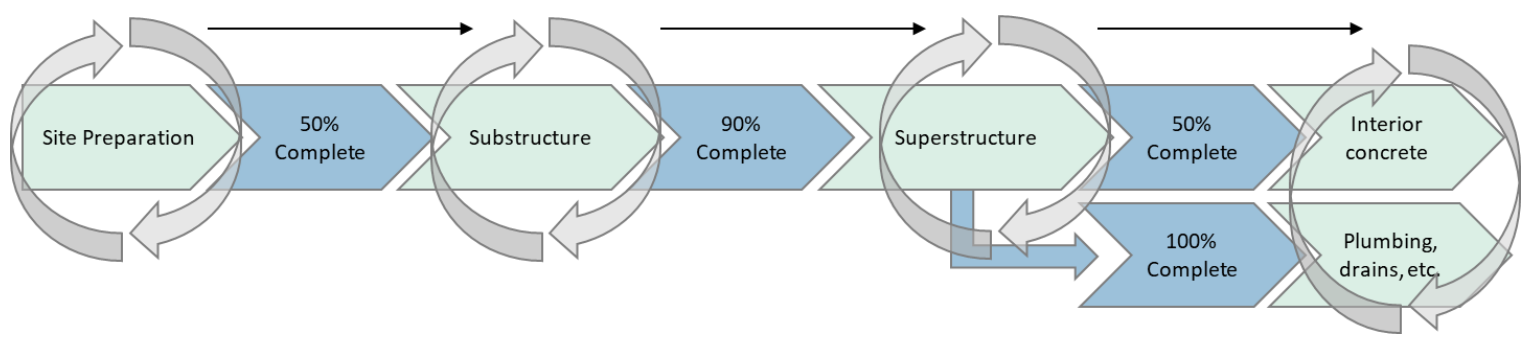

Figure 5. Representation of the subproblem formulation of the optimization problem.

\section{Results}

\section{A. NCET overnight cost and labor hours}

Table 4 contains the results for first-of-a-kind (FOAK) specific overnight cost and FOAK and 10-OAK total direct labor hours. The direct labor hours excluded indirect work such as management and supervision and QA/QC. The results were consistent with what was previously published for the PWR12, LPSR, 
MMNC, and NC-SMR [5], but the BWR results are new, and the detailed scaling behind each cost account is in the supplementary information.

Table 4. Total direct costs and labor hours for each reactor architecture for the FOAK and 10-OAK plants. Note that indirect costs are usually equal to direct costs for FOAK plants.

\begin{tabular}{|l|r|r|r|r|r|}
\hline & $\begin{array}{l}\text { FOAK overnight } \\
\text { direct cost }(\$ / \mathrm{kWe})\end{array}$ & $\begin{array}{l}\text { FOAK direct } \\
\text { labor hours }\end{array}$ & $\begin{array}{l}\text { FOAK direct labor } \\
\text { hours per GWe }\end{array}$ & $\begin{array}{l}\text { 10-OAK direct } \\
\text { labor hours }\end{array}$ & $\begin{array}{l}\text { 10-OAK direct labor } \\
\text { hours per GWe }\end{array}$ \\
\hline PWR12 & 2,300 & $22,000,000[\mathrm{ME}]$ & 19.2 & $13,800,000[\mathrm{BE}]$ & 12.1 \\
\hline LPSR & 2,100 & $17,800,000$ & 21.8 & $11,100,000$ & 10.0 \\
\hline MMNC & 3,400 & $18,500,000$ & 31.8 & $3,800,000$ & 14.4 \\
\hline NC-SMR & 4,200 & $5,100,000$ & 15.4 & $13,100,000$ & 23.8 \\
\hline LM-BWR & 2,300 & $20,800,000$ & 25.0 & $4,500,000$ & 9.7 \\
\hline SM-BWR & 3,400 & $7,300,000$ & & & 15.7 \\
\hline
\end{tabular}

B. Repeatability of GA solution

GAs are stochastic and do not guarantee finding the exact optimum, so to verify the population size and convergence criteria selection were appropriate, the full-scale GA optimized the PWR12-BE plant schedule from ten sets of different random initial populations of size 500,1000, and 1500 . The trajectories of the best individuals in the populations from the ten runs of population 1000 is shown in Figure 6 . Each case followed a very similar convergence path, and the optimal solutions ranged from 7380 months with an average of 76.5 months. The population 500 set ranged from 78-85 with an average of 82.2, and the population 1500 set ranged from 70-81 with average 74.8. All the population 1000 set were within $15 \%$ of the minimum schedule length found of 70 months, and therefore, for the remainder of the reactor architectures, a population of 1000 was a good balance of accuracy and computation time.

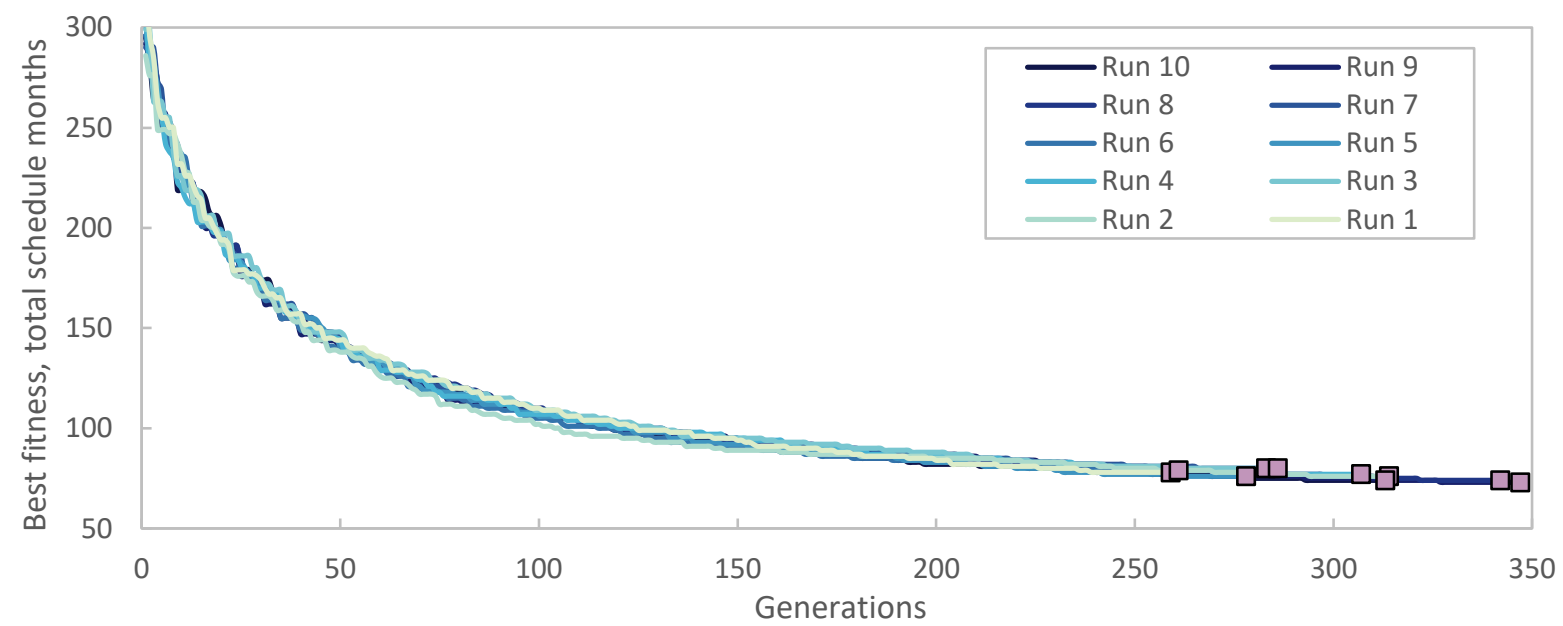

Figure 6. Repeatability of the converged GA solution for the PWR12BE. Population size: $1000 . f$-tolerance and $x$-tolerance: $1 e-3$

The subproblem GA ran ten times starting from one solution of the full-scale $G A$ with four loops through every timestep. The population sizes were ten times the number of decision variables at each timestep. The ten solutions averaged 67 months, ranged from 65-76 months, and all but one case was between 65-68 months. 


\section{Benchmark results: PWR12-BE}

To benchmark the assumptions for the task dependencies and lower-bound constraints that were based on the IAEA and EEEDB data, we compared the resulting staffing profile from an optimization run for the PWR12-BE (10-OAK output from NCET) to data from an IAEA report for a similar reactor architecture. The IAEA published a sample staffing profile for a 72-month PWR construction project which was also the reported construction timeline for the PWR12-BE from EEDB [19,32]. The IAEA reported the data as fractions of a normalized peak, so we scaled the normalized values such that the integral matched the PWR12-BE total labor. Figure 7shows a comparison between the IAEA data and one run of the full-scale $G A$ coupled to the subproblem GA using the lower-bound constraints.

The NCET results followed similar trends to the IAEA data: an early peak dominated by civil work, a later peak driven by electrical and mechanical work, and overlapping electrical and mechanical work. There were some notable differences: the NCET profile was not smooth, and civil work peaked earlier and higher in the NCET results. Many of the tasks were not critical path, so their delay and task lengths were relatively arbitrary in the scope of the schedule optimization problem, and this dynamic allowed for the peaky nature of the staffing profile. In other words, there was no penalty function for month-to-month variation in staffing level as there would be in practice. This same dynamic caused the early civil works peak because construction could begin on every structure after site preparation completed, but the model constraints did not force this work to be levelized to the extent a project planner would. However, the primary figure of merit was the total construction duration as determined by the critical path, and the NCET optimized duration was 71 months compared to the published PWR12-BE duration of 72 months.
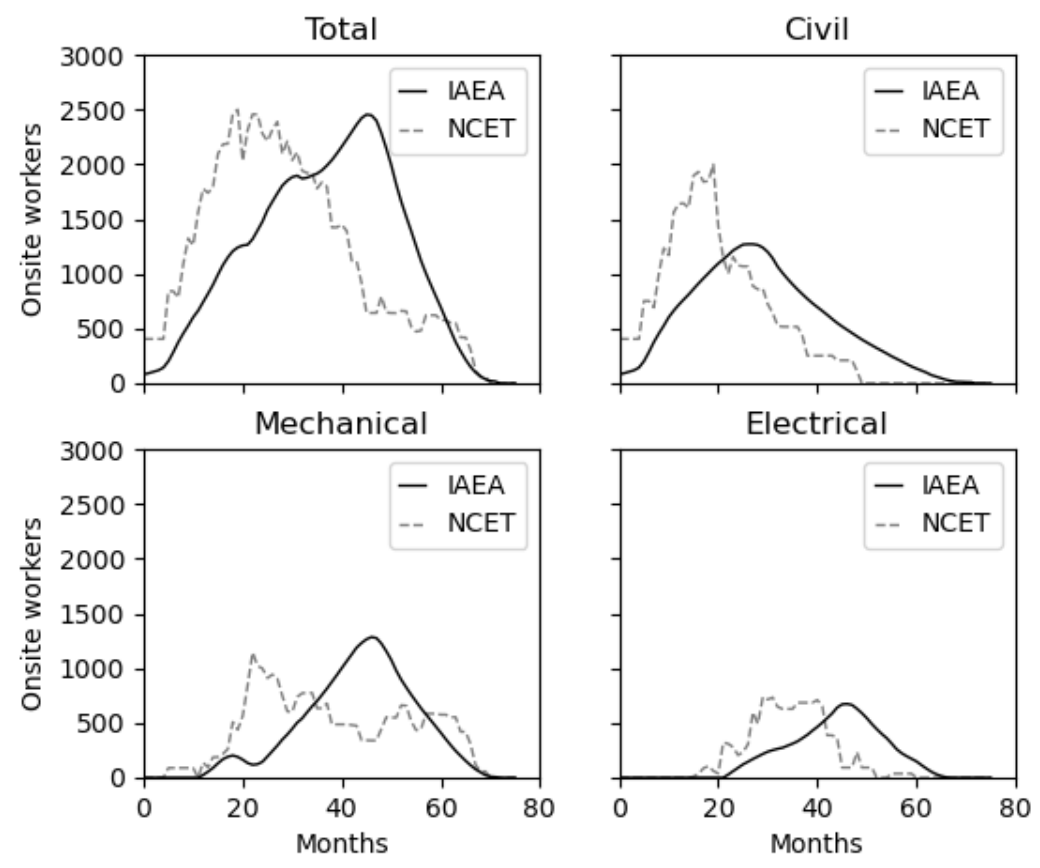

Figure 7. Staffing profile comparison between the IAEA reported data and the resulting staffing profile from an optimization of the PWR12-BE schedule using to lower bound constraints. 
In addition to the qualitative comparison to the IAEA staffing profile, Table 5 contains a quantitative comparison between published construction durations and estimates from this study. For the PWR12 and LM-BWR, the published durations were realized durations from constructed plants, but the others are estimates published by the vendor, and FOAK vendor estimates should be viewed with some scrutiny because of unforeseen construction delays. The NCET duration estimates using the upper bound constraints were all within $15 \%$ of the published durations indicating that the methodology and constraints capture the dynamics of the construction process well. The Pearson correlation coefficient between the datasets was 0.99 , and the root mean square error was 4.3 months.

Table 5. Table of benchmarks with the results from the NCET scheduler compared to other schedule estimates or observations using the upper-bound constraints: max site staffing of 4500 and max monthly labor change of 800.

\begin{tabular}{|l|l|l|}
\hline & Published duration (months) & NCET estimated duration (months) \\
\hline PWR12-BE & $72[19]$ & $64(-11 \%)$ \\
\hline PWR12-ME & $98[19]$ & $100(+2 \%)$ \\
\hline LPSR & 54 (FOAK estimate [10]) & $52(-4 \%)$ \\
\hline MMNC & 36 (NOAK estimate [40]) & $34(-6 \%)$ \\
\hline NC-SMR & Proprietary (estimate) & $+/-15 \%$ \\
\hline LM-BWR & 40 (NOAK,[17]) & $35(-8 \%)$ \\
\hline SM-BWR & 26 (NOAK estimate, [28]) & $29(+12 \%)$ \\
\hline
\end{tabular}

The staffing profiles for each plant revealed insights into each reactor architecture and the dynamics of the modeling methodology. Figure 8 contains the staffing profiles for the six reactor architectures with the 10-OAK person-hours per task, and the upper bound constraints. The profiles with long tails in Figure 8, PWR12, NC-SMR, and SM-BWR, were the result of critical path tasks that were building staffing limit constrained as opposed to peak site staff or labor rate constrained. For these plants, the subproblem GA methodology shifts the less constrained tasks early in the timeline causing the early peak in staffing levels. In practice, a more levelized staffing approach would likely be adopted - lowering the peak.

The other three architectures, LPSR, MMNC, and LM-BWR, all have more triangular shaped profiles. In this case, the architectures were limited by the peak site staff and labor change constraints than the building staffing constraint. This was mostly because they expanded the construction area by using the VHLC to lift modules into place that were fabricated adjacent to the building. 

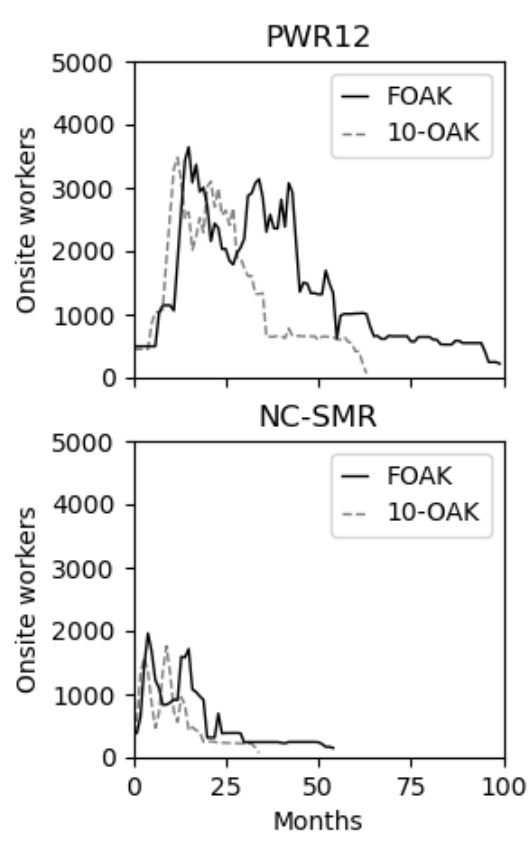

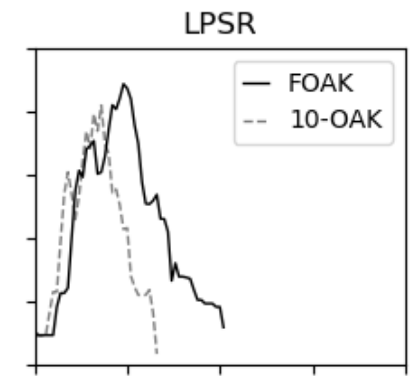

LM-BWR

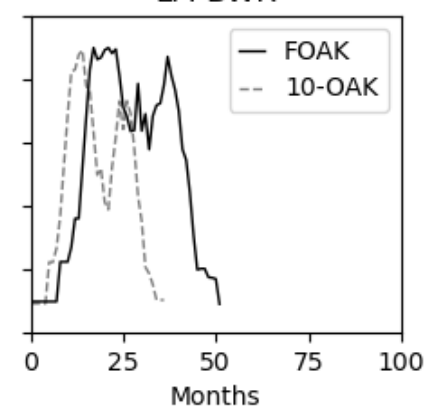

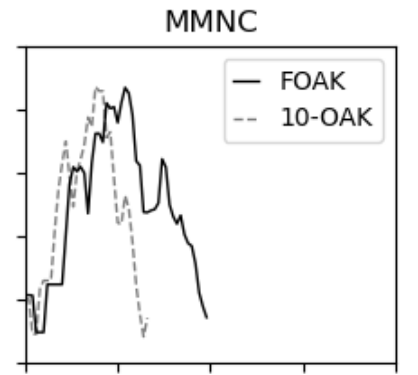

SM-BWR

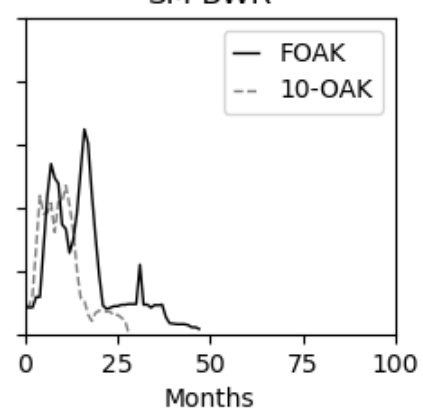

Figure 8. Staffing profiles for six reactor architectures for the FOAK and 10-OAK plant under the upper-bound constraints.

\section{Sensitivity}

The result of the benchmarks suggested that most reactor vendors assumed minimal constraints on peak hiring rates and staffing levels, especially in comparison to the IAEA staffing profile. We examined four variations of the upper and lower bound constraints, and Table 6 summarizes the results. On average, the lower-bound constraints increased the construction duration $23 \%$ for FOAK plants and $28 \%$ for 10-OAK plants. However, the effect was inconsistent across the reactor architectures. The small reactors, NC-SMR and SM-BWR, and the PWR12 were mostly insensitive to the tighter constraints while the LPSR, MMNC, and LM-BWR all experienced significant delays. The PWR12-ME did not experience significant delays partly because its construction time was already longer than the other large plants.

The low total person-hours of work benefitted the small reactors in this regard, and this is a widely accepted advantage of SMRs - that they can be constructed with a limited workforce. The PWR12 plant was insensitive to these constraints because it was more limited by the building staff constraint because it did not use the VHLC to parallelize work onsite and increase the number of usable workers. Here we see a potential disadvantage of the VHLC, if enough workers cannot be hired quickly, adopting this technology may not be worthwhile. In the 12-module embodiment, the MMNC, though generally considered an SMR, did not have this advantage. Table 6 shows that the MMNC was not substantively different from the LPSR, and in fact, the MMNC was more sensitive to these constraints than the LPSR.

We also tested which of the constraints, the maximum site staffing or the maximum monthly labor change, had the greater effect in increasing construction durations. The results in Table 6 show that the 160 -worker monthly change increased construction durations $20 \%$ on average from the 800 -worker limit, and the 2500 -worker peak site staffing increased durations $13 \%$. Therefore, how quickly workers can be recruited and hired was the more limiting factor in this comparison. An Inspector General report on the issues at Watts Bar Unit 2 confirmed that hiring rate and availability of workers was a key 
management and schedule issue [41]. This is a key benefit of multi-site plants where already trained workers can be shifted from unit to unit during sequential construction.

Table 6. FOAK construction duration estimates in months under different constraints. The percentages are the difference relative to the upper bound constraints.

\begin{tabular}{|l|l|l|l|l|}
\hline & $\begin{array}{l}\text { Max site: } 4500 \\
\text { Max change: } 800\end{array}$ & $\begin{array}{l}\text { Max site: 2500 } \\
\text { Max change: } 160\end{array}$ & $\begin{array}{l}\text { Max site: 4500 } \\
\text { Max change: 160 }\end{array}$ & $\begin{array}{l}\text { Max site: 2500 } \\
\text { Max change: } 800\end{array}$ \\
\hline PWR12-ME & 100 & $99(-1 \%)$ & $99(-1 \%)$ & $98(-2 \%)$ \\
\hline LPSR & 52 & $68(+31 \%)$ & $64(+23 \%)$ & $58(+12 \%)$ \\
\hline MMNC & 50 & $72(+44 \%)$ & $70(+40 \%)$ & $63(26 \%)$ \\
\hline NC-SMR & 55 & $57(+4 \%)$ & $57(+4 \%)$ & $58(+5 \%)$ \\
\hline LM-BWR & 52 & $79(+52 \%)$ & $75(+44 \%)$ & $71(+37 \%)$ \\
\hline SM-BWR & 42 & $43(+2 \%)$ & $49(+17 \%)$ & $43(+2 \%)$ \\
\hline Average & $\mathbf{2 2 \%}$ & $\mathbf{2 0 \%}$ & $\mathbf{1 3 \%}$ \\
\hline
\end{tabular}

The smaller plants, SM-BWR and NC-SMR, did not hit the peak staffing limitation in either the upper bound or lower bound case as shown in Figure 9. In contrast, the large plants using the VHLC, LPSR, MMNC, and LM-BWR, were near both peak staffing limitations. The AP1000 construction site in Vogtle had difficulty hiring enough skilled workers, and the need for a smaller workforce is a purported advantage of SMRs, and this result confirms this advantage [42].

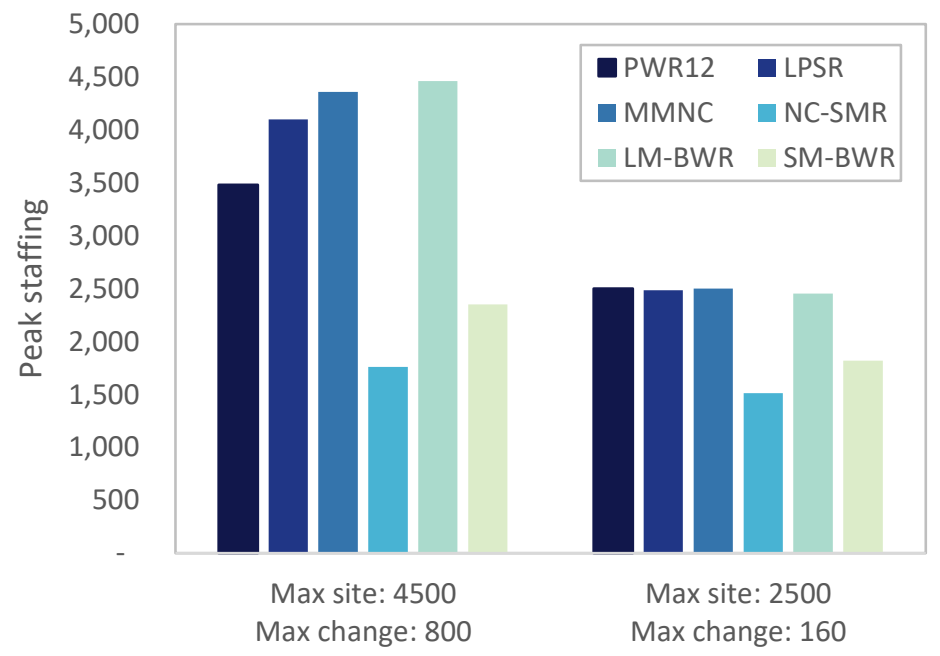

Figure 9. 10-OAK peak staffing for the two different constraint cases and the six reactor architectures

\section{E. Indirect cost savings from modularization}

Shorter construction schedules result in lower IDC and lower indirect costs due to shorter construction management periods and home office engineering services. While the former is intuitive, that latter requires some explanation. Taking the fixed direct costs output from NCET for the FOAK LPSR, and varying the construction duration, we see the varying indirect cost outputs in Figure 10. Indirect site material costs were predominantly construction tools and equipment, so a long construction duration required less staffing and in turn less tooling. Indirect site labor costs were predominantly temporary construction services such as roads, parking, laydown area, and electrical supply which were fixed costs. Indirect factory costs were largely home office engineering services and field job supervision, so these 
costs scale linearly with the construction duration. Therefore, durations longer than 60 months for the FOAK LPSR start to see significant indirect costs.

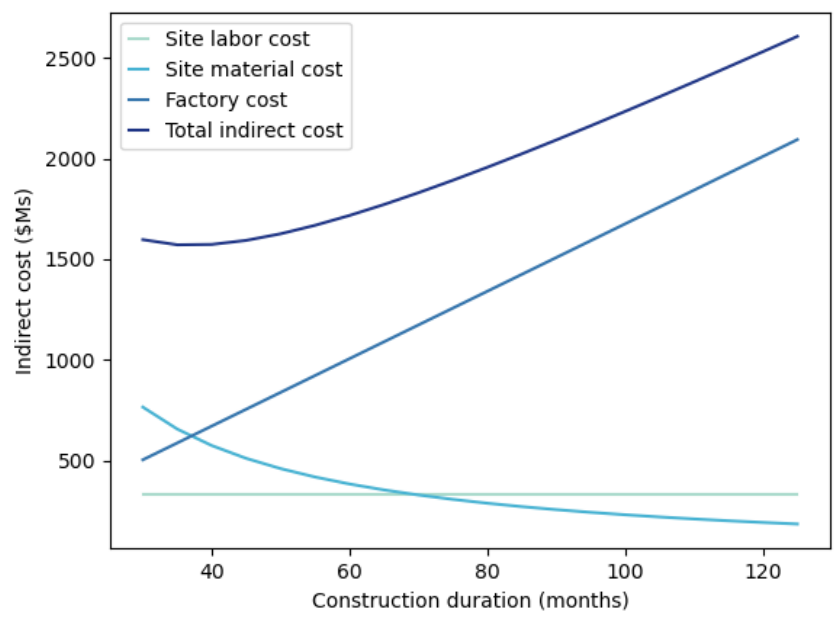

Figure 10. LPSR FOAK indirect costs for fixed direct costs and varying construction durations.

We estimated the reduction in IDC and indirect costs from the shorter construction schedules enabled by the VHLC for the 10-OAK LPSR and the NC-SMR. To do this, we removed the modulization of the SSCs and parallelization of site work in two stages: system modules and structural modules. System modules included the reactor pressure vessel, internals, and control system; safeguard systems such as safety injection and heat removal; waste processing equipment; the turbine generator system; and the service air, water, and steam systems. These are systems that can be broken down into factory assembled modules, shipped to the site, and installed via crane. Structural modules included the interior and superstructure concrete walls for the reactor and auxiliary buildings.

The structural modules had a greater impact on the construction duration than the system modules. The results in Table 7 show that removing the system modules extended the construction duration $29-44 \%$ and removing all modules increased the duration $135-169 \%$. Interestingly, the impact of eliminating all modules was greater for the SMR, and this was because the NC-SMR design is a tall, thin building. Eliminating the VHLC and labor parallelization severely constricts access because the work area is small, and this extends the construction duration. In general, structural modules reduced construction times $39-52 \%$, and system modules were a further $22-31 \%$ reduction.

Table 7. Construction duration in months for the NC-SMR and LPSR with and without system modules of mechanical systems and structural modules of civil structures.

\begin{tabular}{|l|r|l|l|}
\hline & $\begin{array}{l}\text { Fully } \\
\text { modularized }\end{array}$ & $\begin{array}{l}\text { No system } \\
\text { modules }\end{array}$ & No modules \\
\hline NC-SMR & 35 & $45(+29 \%)$ & $94(+169 \%)$ \\
\hline LPSR & 34 & $49(+44 \%)$ & $80(+135 \%)$ \\
\hline
\end{tabular}

Assuming a $8 \%$ interest rate and adopting the discrete interest accumulation equation of Champlin [2]:

$$
I D C=\frac{(1+i)^{1+N}-(1-i)}{i N}
$$


Where $N$ is the number of months and $i$ is the interest rate. Figure 11 shows the financial consequences of the timeline extensions. For each case, the IDC was greater for the NC-SMR than the LPSR in absolute specific costs, but relative to their overnight costs they were similar. This was because the 10-OAK NCSMR overnight direct cost was $\$ 2,800 / \mathrm{kWe}$ and the LPSR overnight cost was $\$ 1,700 / \mathrm{kWe}$. The no system modules cases increased the IDC by 25 and $38 \%$, and the no modules cases increased the IDC by $168 \%$ and $128 \%$ for the NC-SMR and LPSR respectively. In total, modularization lowered indirect costs $\$ 2,300 / \mathrm{kWe}$ for the NC-SMR and $\$ 900 / \mathrm{kWe}$ for the LPSR. Structural modularization via the VHLC with parallel construction work areas greatly reduces financing costs for nuclear projects but increases the risk of insufficient worker and material supply.
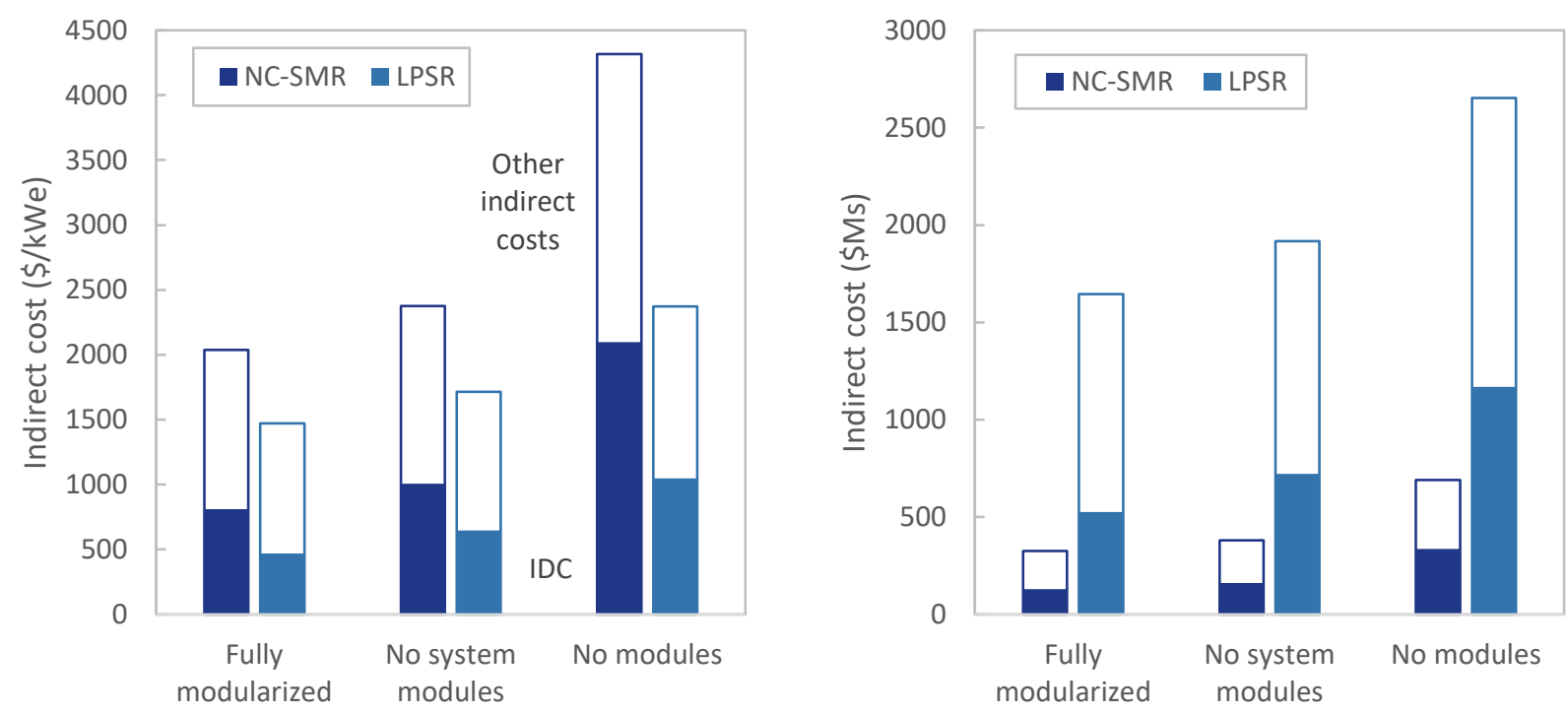

Figure 11. Indirect costs for the 10-OAK NC-SMR and LPSR under different modularization assumptions including both the IDC (colored bars) and other indirect costs (white bars). Left: indirect costs per unit capacity, right: absolute indirect costs. Discount rate: $8 \%$

It should be noted the owner's cost is also dependent on the indirect cost, and this makes meeting the targeted construction schedule an even more significant part of hitting the target total project cost.

\section{Conclusions}

This study presents a simplified methodology for estimating the construction duration of nuclear projects. Using the labor hours associated with 226 tasks, a two-step genetic algorithm builds a Gantt chart using previously defined task dependencies that minimizes the total construction time.

Benchmarking this process against seven published construction durations showed good agreement with the model estimates.

Applying the model to six different reactor architectures revealed several insights. Reactor vendor estimates assume minimal constraints on labor acquisition when in practice this may be a limiting condition, but the smaller reactors were insensitive to these constraints such as the NC-SMR and SMBWR. The limited workforce required to construct these plants was a great advantage, and this study confirmed what was expected of small reactors. The 12-module MMNC did not have this advantage, but NuScale has begun advertising 4- and 6-module plants which may see some of this insensitivity. The monthly deployable worker rate was a more limiting constraint than the limit on the peak site staffing. 
VHLC technology coupled with parallel construction work areas reduced the sensitivity to the building staffing constraint and reduced total construction time. In the case of the LPSR and NC-SMR, structural modularization reduced construction $45 \%$ on average, and system modularization reduced construction duration $26 \%$ on average. However, if not enough workers can be hired in a timely fashion, adopting this technology may not be worthwhile.

The small reactors are expected to be more sensitive to the modularization assumptions because their compact footprint constricts access for construction. At the same time, the total absolute cost of delays was significantly smaller and poses less financial risk.

This study excluded several elements of the construction schedule including quality assurance and control, startup, and regulatory activities. The methodology we present analyzes a best case scenario for nuclear construction projects, and a best case scenario has been rare in recent decades in the US and Europe. Therefore, future work should include a risk assessment of delay probabilities for the GA-built Gantt charts including supply chain delays, construction delays, rework, and regulatory changes.

Different reactor architectures may have different exposure to risk and resulting cost escalations.

\section{Acknowledgements}

This work has been supported by Fortum, www.fortum.com. The authors thank Prof. Jacopo Buongiorno (MIT) and Prof. Oli de Weck (MIT) for reviewing the paper and providing comments.

\section{References}

[1] Carelli MD, Ingersoll DT. Handbook of small modular nuclear reactors. 2nd ed. 2020.

[2] Champlin PA. Techno-economic evaluation of cross-cutting technologies for cost reduction in nuclear power plants. Massachusetts Institute of Technology, 2018.

[3] Nuclear Energy Agency. Unlocking Reductions in the Construction Costs of Nuclear. Paris: 2020.

[4] Third Way. Advanced Nuclear Map 2019. https://www.thirdway.org/graphic/2019-advancednuclear-map (accessed July 14, 2021).

[5] Stewart WR, Shirvan K. Capital Cost Estimation for Advanced Nuclear Power Plants. Renewable and Sustainable Energy Reviews 2021; In Press.

[6] Mignacca B, Locatelli G. Economics and finance of Small Modular Reactors: A systematic review and research agenda. Renewable and Sustainable Energy Reviews 2020;118:109519. https://doi.org/10.1016/J.RSER.2019.109519.

[7] Berthélemy M, Escobar Rangel L. Nuclear reactors' construction costs: The role of lead-time, standardization and technological progress. Energy Policy 2015;82:118-30. https://doi.org/10.1016/J.ENPOL.2015.03.015.

[8] Lovering JR, Yip A, Nordhaus T. Historical construction costs of global nuclear power reactors. Energy Policy 2016;91:371-82. https://doi.org/10.1016/J.ENPOL.2016.01.011.

[9] Ingersoll E, Gogan K, Herter J, Foss A. The ETI Nuclear Cost Drivers Project: Full Technical Report. 2020. 
[10] Dominion Energy Inc., Bechtel Power Corporation, TLG Inc, MPR Associates. Study of Construction Technologies and Schedules, O\&M Staffing and Cost, Decommissioning Costs and Funding Requirements for Advanced Reactor Designs. 2004.

[11] Maronati G, Petrovic B, van Wyk JJ, Kelley MH, White CC. Impact of testing activities on small modular reactor total capital investment cost. International Conference on Nuclear Engineering, Proceedings, ICONE, vol. 2, American Society of Mechanical Engineers (ASME); 2016. https://doi.org/10.1115/ICONE24-60675.

[12] Maronati G, Petrovic B, Ferroni P. Assessing I2S-LWR economic competitiveness using systematic differential capital cost evaluation methodology. Annals of Nuclear Energy 2020;145:106202. https://doi.org/10.1016/J.ANUCENE.2018.05.057.

[13] Energy Impact Center. CONSTRUCTION | Open100 2020. https://www.open100.com/construction (accessed December 15, 2021).

[14] Abdulla A, Azevedo IL, Morgan MG. Expert assessments of the cost of light water small modular reactors. Proceedings of the National Academy of Sciences of the United States of America 2013;110:9686-91. https://doi.org/10.1073/PNAS.1300195110/-/DCSUPPLEMENTAL.

[15] Lloyd CA, Roulstone A. A methodology to determine SMR build schedule and the impact of modularisation. International Conference on Nuclear Engineering, Proceedings, ICONE 2018;1. https://doi.org/10.1115/ICONE26-81550.

[16] Peterson PF, Zhao H, Petroski R. Metal And Concrete Inputs For Several Nuclear Power Plants. 2005.

[17] 柏崎刈羽原子力発電所の設備概要 - 新潟県ホームページ n.d. https://www.pref.niigata.lg.jp/sec/genshiryoku/kk-plant.html (accessed December 26, 2021).

[18] Inoue T, Miura J, Murayama K. Hitachi's experience and achievements in ABWR construction. International conference on global environment and advanced nuclear power plants, Kyoto: 2003.

[19] United Engineers and Constructors Inc. Phase 9 update (1987) report for the Energy Economic Data Base Program EEDB-IX. U.S. Department of Energy: 1988. https://doi.org/10.2172/7227212.

[20] Westinghouse Electric Corporation. Status report 81 - Advanced Passive PWR (AP 1000). 2011.

[21] Westinghouse Electric Corporation. NRC: Package ML083230868 - Westinghouse AP1000 Design Control Document Rev. 17 2009. https://www.nrc.gov/docs/ML0832/ML083230868.html (accessed December 26, 2021).

[22] NuScale Power LLC. Application Documents For The NuScale Design 2020. https://www.nrc.gov/reactors/new-reactors/smr/nuscale/documents.html (accessed December 26, 2021).

[23] Colbert C. Update to Information provided at MIT Advanced Reactor Cost Workshop. 2018.

[24] SMR LLC. SMR-160 A Safe and Secure Nuclear Energy Future for Ukraine. 2017. 
[25] Singh K. SMR-160: A Walk-away Safe Small Modular Nuclear Reactor. Washington D.C.: 2013.

[26] GE-Hitachi. Status report 97 - Advanced Boiling Water Reactor (ABWR). 2011.

[27] GE-Hitachi. ABWR | NRC.gov 1997. https://www.nrc.gov/reactors/new-reactors/designcert/abwr.html\#dcd (accessed December 26, 2021).

[28] GE-Hitachi, Hitachi GE Nuclear Energy. Status Report - BWRX-300. 2019.

[29] GE-Hitachi. Revision 0, BWRX-300 Advanced Civil Construction and Design Approach. 2021.

[30] Bertram N, Fuchs S, Mischke J, Palter R, Strube G, Woetzel J. Modular construction: From projects to products. 2019.

[31] Japan Atomic Energy Agency. Location, construction, operation, and maintenance of nuclear power plants. 2008.

[32] IAEA. Manpower Development for Nuclear Power. 1980.

[33] Wall MB. A genetic algorithm for resource-constrained scheduling. Massachusetts Institute of Technology, 1996.

[34] World Nuclear News. UAE's fourth power reactor under construction. World Nuclear News 2015. https://www.world-nuclear-news.org/NN-UAEs-fourth-power-reactor-under-construction0209155.html (accessed December 20, 2021).

[35] EDF. Hinkley Point C Application Summary Document. 2011.

[36] IAEA. Construction Technologies for Nuclear Power Plants. Vienna: 2011.

[37] Maronati G, Petrovic B, van Wyk JJ, Kelley MH, White CC. Impact of testing activities on small modular reactor total capital investment cost. International Conference on Nuclear Engineering, Proceedings, ICONE 2016;2. https://doi.org/10.1115/ICONE24-60675.

[38] Nawara TM. Resource constrained scheduling problem at U.S. Naval Shipyards. Massachusetts Institute of Technology, 2013.

[39] Blank J, Deb K. Pymoo: Multi-Objective Optimization in Python. IEEE Access 2020;8:89497-509. https://doi.org/10.1109/ACCESS.2020.2990567.

[40] NuScale SMR. Status Report - NuScale SMR. 2020.

[41] Tennessee Valley Authority Office of the Inspector General. Inspection Report: WATTS BAR NUCLEAR PLANT UNIT 2 PROJECT SET-UP AND MANAGEMENT ISSUES AFFECTED COST AND SCHEDULE. 2012.

[42] Trump Administration Issues Shock Visa Denial to Canadian Members. The Electrical Worker Online 2018. http://www.ibew.org/articles/18ElectricalWorker/EW1810/north49a.1018.html (accessed January 2, 2022). 